\title{
Pre-Anesthesia Extracorporeal Membrane Oxygenation in Two Lung Transplant Recipients with Severe Pulmonary Hypertension
}

\author{
Liu Minqiang, ${ }^{1}$ Gao Hong, ${ }^{2}$ Chen Jingyu $\left(\mathbb{D},{ }^{3}\right.$ Wang Yanjuan, ${ }^{2}$ Xu Bo, ${ }^{2}$ Wang Guilong, \\ Wu Qiang, ${ }^{1}$ and Hu Chunxiao (iD $^{2}$ \\ ${ }^{1}$ Department of Anesthesiology, The Third People's Hospital of Shenzhen, No. 29 Bulan Road, Longgang District, Shenzhen, \\ Guangdong 518112, China \\ ${ }^{2}$ Department of Anesthesiology, Wuxi People's Hospital, 299 Qingyang Road, Liangxi District, Wuxi, Jiangsu 214023, China \\ ${ }^{3}$ Department of Lung Transplantation Center, Wuxi People's Hospital, 299 Qingyang Road, Liangxi District, Wuxi, \\ Jiangsu 214023, China \\ Correspondence should be addressed to Hu Chunxiao; huchunxiao91211@163.com
}

Received 14 October 2019; Accepted 14 November 2019; Published 12 February 2020

Academic Editor: Michael S. Firstenberg

Copyright ( $) 2020$ Liu Minqiang et al. This is an open access article distributed under the Creative Commons Attribution License, which permits unrestricted use, distribution, and reproduction in any medium, provided the original work is properly cited.

Extracorporeal membrane oxygenation (ECMO) is a widely used cardiopulmonary support method that is usually implemented after anesthesia during the period of lung transplantation (LTx). In severe pulmonary arterial hypertension (PAH) patients, however, anesthesia induction is a high-risk phase and can result in severe cardiorespiratory failure. Herein, we describe two severe PAH patients who received ECMO support before anesthesia and whose preoperative evaluations indicated that the risk was too high to safely survive the anesthesia induction period before LTx. The strategy was successful, and in both patients, hemodynamics was stable and no ECMO-related complications occurred.

\section{Introduction}

Severe pulmonary arterial hypertension (PAH) has been defined as mean pulmonary arterial pressure (mPAP) greater than $35 \mathrm{mmHg}$ [1]. Patients with severe PAH are usually in a critical condition with right heart function on the verge of failure, and anesthesia management in these patients is challenging. During the induction period of anesthesia, the associated sudden sympathetic nerve depression and change in respiratory pattern can aggravate right heart dysfunction and cause severe chest tightness, dyspnea, and hypotension, and it can even lead to pulmonary hypertension crisis [2]. In an effort to avoid uncontrolled hemodynamic deterioration, the successful application of extracorporeal membrane oxygenation (ECMO) before general anesthesia induction has previously been reported [3]. To improve the safety of these patients during perioperative period, we decided to perform ECMO assistance before anesthesia induction in severe $\mathrm{PAH}$ patients whose mPAP was greater than $50 \mathrm{mmHg}$, or pulmonary artery systolic pressure (PASP) greater than
$100 \mathrm{mmHg}$, and cardiac index (CI) less than $2 \mathrm{~L} / \mathrm{min} / \mathrm{m} \mathrm{[2].}$ We hope that this strategy will help some patients to survive the anesthesia induction period stably.

\section{Case Presentation}

The strategy described below was approved by the Medical Ethics Committee of Wuxi People's Hospital, Nanjing Medical University, Wuxi, Jiangsu, China (approval number KYLLH2018022). Written informed consent was obtained from each patient before anesthesia. Both patients were in a state of cardiorespiratory decompensation despite maximum pharmacological therapy.

2.1. Case 1. A 22-year-old male was diagnosed as idiopathic pulmonary hypertension. Preoperative right cardiac catheterization revealed $\mathrm{mPAP}$ increased to up to $81 \mathrm{mmHg}$, CI dropped down to $1.98 \mathrm{~L} / \mathrm{min} / \mathrm{m}^{2}$ (Figures $1(\mathrm{a})$ and $1(\mathrm{~b})$ ), and B-type natriuretic peptide (BNP) rose to $1134 \mathrm{pg} / \mathrm{ml}$. 


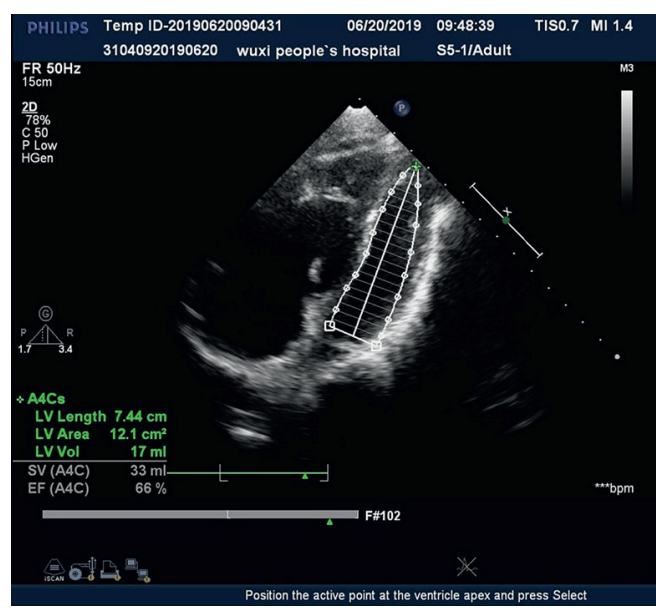

(a)

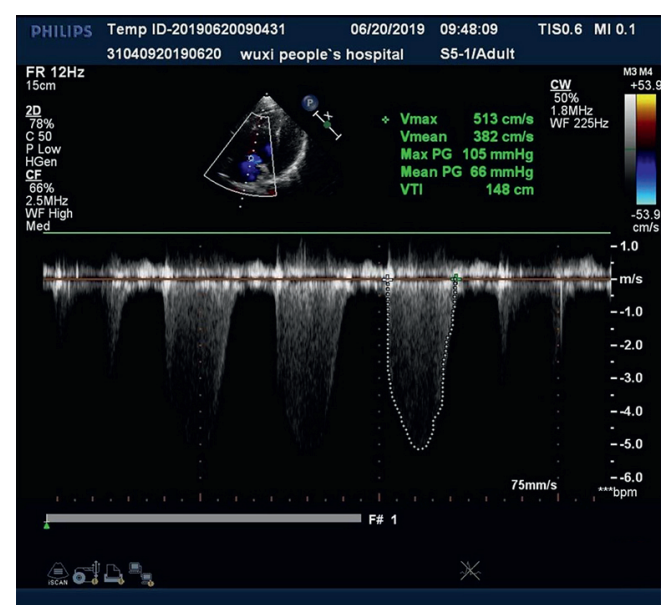

(b)

Figure 1: (a) Transthoracic echocardiography in patient 1 depicted substantial enlargement of the right atrium and right ventricle, and widening of the pulmonary artery. (b) Pulmonary artery systolic pressure increased to up to $120 \mathrm{mmHg}$.

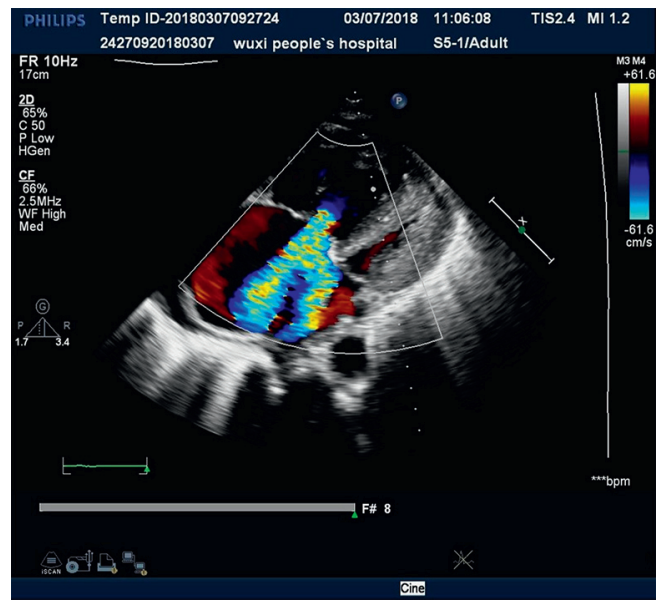

(a)

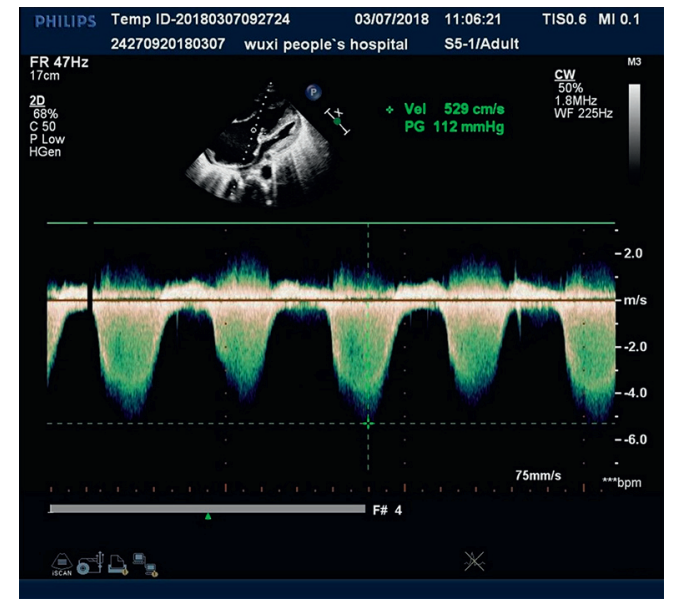

(b)

FIgURE 2: (a) Transthoracic echocardiography in patient 2 depicted obvious widening of the right atrium and right ventricle and severe regurgitation of the tricuspid valve. The pulmonary artery was distinctly broadened. (b) Pulmonary artery systolic pressure increased to up to $118 \mathrm{mmHg}$.

2.2. Case 2. A 59-year-old female diagnosed with diopathic interstitial pneumonia. Preoperative right cardiac catheterization revealed $\mathrm{mPAP}$ rose up to $67 \mathrm{mmHg}$, CI fallen to $1.82 \mathrm{~L} / \mathrm{min} / \mathrm{m}^{2}$ (Figures $2(\mathrm{a})$ and $2(\mathrm{~b})$ ), and BNP ascended to $5328 \mathrm{pg} / \mathrm{ml}$.

After patient admission, the intravenous medication of $\mathrm{PAH}$ was maintained via a catheterization of the right internal jugular vein obtained in the intensive care unit. $\mathrm{Pa}-$ tients were kept awake, a half sitting position was utilized and peripheral arterial access was secured via a 22-G arterial puncture needle on the right radial hand. After local anesthesia took effect, a dose of heparin $50 \mathrm{U} / \mathrm{kg}$ was injected, and a venoarterial extracorporeal membrane oxygenator was implanted via a catheter from the right femoral artery up to the descending aorta for perfusion, and a second cannula from the right femoral vein to below the junction between the inferior vena cava and the right atrium for drainage, which were monitored with transesophageal echocardiography. During the period of ECMO bypass, the flow rate was adjusted within 1.5-3.0 L/min based on the patient's hemodynamic and oxygenation status, and the activated clotting time was monitored every $2 \mathrm{~h}$, and maintained for $160-200 \mathrm{~s}$ by the administration of heparin $10-20 \mathrm{U} / \mathrm{kg}$ when necessary. In both patients, cardiopulmonary function largely stabilized within approximately 5 to $10 \mathrm{~min}$ with a small dose of infused norepinephrine; the preoperative therapy of PAH was removed, and general anesthesia induction began with an injection of midazolam, etomidate, sufentanil, and cis-atracurium. A Swan-Ganz catheter was then inserted via the right internal jugular vein to continuously monitor central venous pressure, mPAP, CI, systemic vascular resistance, and pulmonary vascular resistance. 
Bedside electrocardiography monitoring indicated that mPAP decreased by approximately $20-25 \mathrm{mmHg}$ and CI rose to $>2 \mathrm{~L} / \mathrm{min} / \mathrm{m}$ [2]. During the surgery, both patients remained hemodynamically stable, and arterial saturation was sufficient. In Case 1, the bilateral LTx completed successfully, mPAP was maintained at approximately $35 \mathrm{mmHg}$, and ECMO support was continued for $24 \mathrm{~h}$ after the surgery. On the other hand, ECMO was discontinued at the end of the surgery in Case 2 because only an unilateral LTx (the right side) was carried out and mPAP rapidly dropped down to $25 \mathrm{mmHg}$ around. Both patients recovered without ECMO-associated complications, and neither exhibited primary graft dysfunction. In both patients, pulmonary artery systolic pressure decreased to $35-39 \mathrm{mmHg}$, and CI and BNP were at normal levels 3 months after the surgery. Cardiopulmonary function had recovered well in both patients at a 6-month follow-up time-point.

\section{Discussion}

The perioperative application of ECMO can effectively alleviate $\mathrm{PAH}$, reduce right ventricular afterload, and treat right heart failure; thus, this supplementary therapy is often needed in severe PAH patients during LTx [4]. It has been reported that patients with severe $\mathrm{PAH}$ associated with lung disease usually had serious right ventricular insufficiency, and that survival was very poor [5]. Moreover, with the inhibitory effects of anesthetics on myocardium and the change in ventilation mode to positive pressure ventilation, pulmonary vascular resistance and ejection resistance of the right heart increase sharply in patients with severe $\mathrm{PAH}$, and right heart dysfunction can occur rapidly, followed by pulmonary flow decline, left ventricular preload and output decrease, systemic hypotension, and even cardiac arrest $[2,6]$. The above-mentioned studies indicate that the induction period of general anesthesia is potentially fatal in patients with severe $\mathrm{PAH}$, and it is very challenging for anesthesiologists to help these patients to undergo anesthesia induction smoothly.

We have described two critical patients with severe $\mathrm{PAH}$, both of whom were with right cardiac failure primary or secondary to respiratory failure and had exhibited rapid deterioration of their disease requiring urgent LTx. Preanesthesia assessment suggested that their cardiopulmonary condition was too bad to tolerate direct anesthesia. However, it was noted that it had been reported that ECMO can occasionally be established with local anesthesia and light sedation before general anesthesia induction, to prevent hemodynamic instability [7]. To avoid further deterioration resulting in cardiorespiratory arrest, we managed ECMO bypass while the patients were still awake. With the assistance of ECMO, pulmonary vascular resistance decreased in both patients, but CI increased markedly, and this contributed to good conditions for the smooth implementation of anesthesia and the subsequent surgery.

In summary, the above-described procedure represents an unconventional strategy involving the provision of ECMO support before anesthesia induction in patients with severe PAH to avoid hemodynamic deterioration. More cases, case series, or comparative studies are needed to evaluate the outcomes associated with this approach, and a well-constructed evaluation scale such as one incorporating the limits of mPAP and CI should also be adopted to assess postoperative complications.

\section{Conflicts of Interest}

The authors have no conflicts of interest.

\section{Acknowledgments}

Thanks to Editage of Cactus (https://support.online.editage. $\mathrm{com} /$ ) for the English edit in this paper.

\section{References}

[1] A. Girard, S. Jouneau, C. Chabanne et al., "Severe pulmonary hypertension associated with COPD: hemodynamic improvement with specific therapy," Respiration, vol. 90, no. 3, pp. 220-228, 2015.

[2] D. McGlothlin, N. Ivascu, and P. M. Heerdt, "Anesthesia and pulmonary hypertension," Progress in Cardiovascular Diseases, vol. 55, no. 2, pp. 199-217, 2012.

[3] K. Hoetzenecker, S. Schwarz, M. Muckenhuber et al., "Intraoperative extracorporeal membrane oxygenation and the possibility of postoperative prolongation improve survival in bilateral lung transplantation," The Journal of Thoracic and Cardiovascular Surgery, vol. 155, no. 5, pp. 2193-2206, 2018.

[4] M.-T. Tsai, C.-H. Hsu, C.-Y. Luo, Y.-N. Hu, and J.-N. Roan, "Bridge-to-recovery strategy using extracorporeal membrane oxygenation for critical pulmonary hypertension complicated with cardiogenic shock," Interactive CardioVascular and Thoracic Surgery, vol. 21, no. 1, pp. 55-61, 2015.

[5] R. G. Pai, P. Varadarajan, N. Kapoor, and R. C. Bansal, “Aortic valve replacement improves survival in severe aortic stenosis associated with severe pulmonary hypertension," The Annals of Thoracic Surgery, vol. 84, no. 1, pp. 80-85, 2007.

[6] A. C. Shukla and M. C. Almodovar, "Anesthesia considerations for children with pulmonary hypertension," Pediatric Critical Care Medicine, vol. 11, pp. S70-S73, 2010.

[7] M. M. Hoeper, R. L. Benza, P. Corris et al., "Intensive care, right ventricular support and lung transplantation in patients with pulmonary hypertension," European Respiratory Journal, vol. 53, no. 1, Article ID 1801906, 2019. 